\title{
Removal of a Large Right Atrial Thrombus with AngioVac Device
}

\author{
Gliceida Galarza Fortuna, $M D^{1}$, Francisco Ujueta, $M D, M S^{1}$, Matthew Lozier, $M D^{2}$, Ivan \\ A. Arenas, MD, PhD, FACC, FASE ${ }^{2}$ and Angelo La Pietra, $M D^{3}$
}

${ }^{1}$ Department of Internal Medicine, Mount Sinai Medical Center, Miami Beach, Florida, USA

${ }^{2}$ Columbia University Division of Cardiology at Mount Sinai Medical Center, 4300 Alton Road, Miami Beach, Florida, USA

${ }^{3}$ Department of Thoracic and Cardiovascular Surgery, Mount Sinai Medical Center, Miami Beach, Florida, USA

\section{Introduction}

Cancer patients often require placement of long-term central venous catheters for chemotherapy and the administration of other intravenous therapies. Catheter-related thrombosis is a known complication of implantable venous access ports, and has an approximate incidence of 3.8\%; regardless of this, primary thromboprophylaxis is not currently recommended in this patient population [1]. Clinical presentation of catheter-related thrombosis varies greatly, ranging from asymptomatic to pulmonary embolism [2]. Therapeutic options for this complication often involve surgical embolectomy and systemic anticoagulation. Recently, percutaneous vacuum-assisted thrombectomy utilizing the AngioVac aspiration system has been introduced as a feasible option. We present a case of a 51-year-old male patient with a pertinent history of rectal cancer who was found to have superior right atrial wall mobile mass successfully treated with AngioVac thrombectomy.

\section{Case Presentation}

A 51-year-old male presented to the emergency department for altered mental status, progressive shortness of breath, and a non-productive cough for three days. Due to altered mentation and tangential speech, history was obtained from the patients' partner at bedside.

Upon arrival, the patient was noted to have a heart rate of 96 beats per minute as well as tachypnea at 24 breaths per minute $\mathrm{O}_{2}$ saturation was $94 \%$ on 4 liters oxygen by nasal cannula). Physical examination was significant for bilateral rhonchi and expiratory wheezing; however, no audible murmurs, skin, nail, or eye lesions were observed. D-dimer was elevated at $6.28 \mu \mathrm{g} / \mathrm{mL}$ FEU and electrocardiogram demonstrated normal sinus rhythm with an incomplete right bundle branch block (Figure 1). Pulmonary computed tomography angiography was remarkable for scattered areas of hypo-attenuation within the segmental bilateral lower lobe pulmonary arteries, thought to be thromboembolic in nature. Subsequent transthoracic echocardiogram (TTE) revealed a large mass originating at the inferior vena cava (IVC) ostium and superior right atrial wall. The mass elongated into a highly mobile strand extending over $5.0 \mathrm{~cm}$ and prolapsing into the right ventricle during diastole (Figure 2 ).

\section{Medical History}

The patient's medical history was notable for HIV with ongoing antiretroviral therapy (consisting of elvitegravir, cobicistat, emtricitabine, and tenofovir alafenamide) and anal carcinoma with chemo-radiation six weeks prior to presentation. A right-sided chemo-port was placed by the trans-jugular approach six months prior for chemotherapy administration. The patient denied history of intravenous drug use, poor dentition/dental issues, unrepaired cyanotic congenital heart defects, or previous placement of artificial heart valves/intracardiac devices.

\section{Differential diagnosis}

The presence of a large and highly mobile structure within the right atrium, in a patient with an intravascular device and known history of malignancy raises concern for venous thromboembolic events such as intra-cardiac thrombus versus nonbacterial thrombotic endocarditis. Additional differential diagnoses include infective endocarditis, metastatic disease to the heart, and primary cardiac tumor.

\section{Investigations}

For further characterization, the patient underwent a transesophageal echocardiogram (TEE), revealing a large ho-

*Corresponding author: Ivan A. Arenas MD, PhD, Columbia University Division of Cardiology, Mount Sinai Medical Center. 4300 Alton Road, Suite \# 2070A, Miami Beach, FL 33140, USA, Tel: +1 305674 2260; Fax: +1 305674 2169, E-mail: ivan.arenas@msmc.com

Accepted: December 29, 2020

Published online: December 31, 2020

Citation: Galarza Fortuna G, Ujueta F, Lozier M, et al. (2020) Removal of a Large Right Atrial Thrombus with AngioVac Device. J Cardiothorac Surg Ther 4(1):75-78 


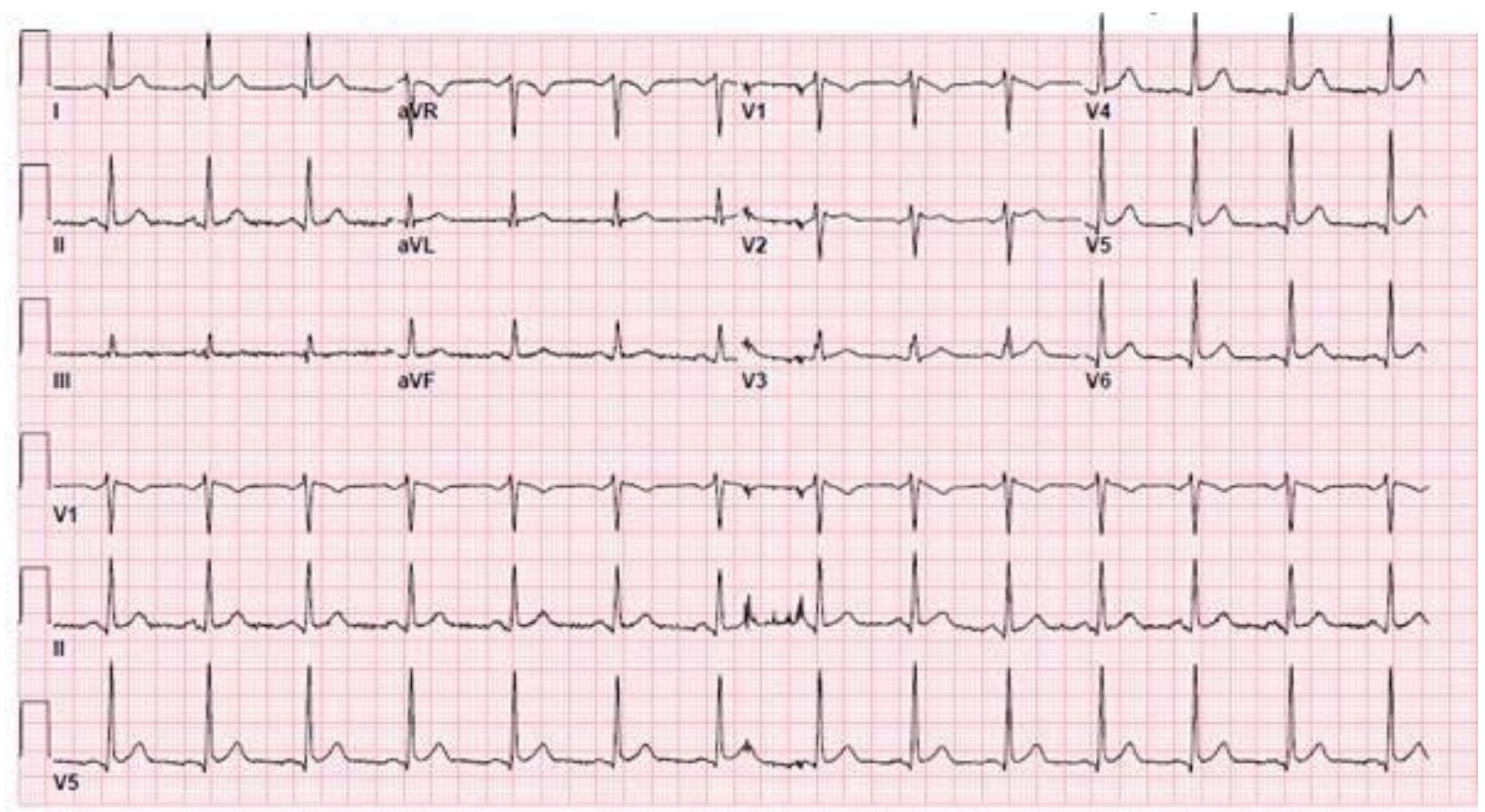

Figure 1: Electrocardiogram demonstrating normal sinus rhythm with an incomplete right bundle branch block (RSR' pattern in V1).
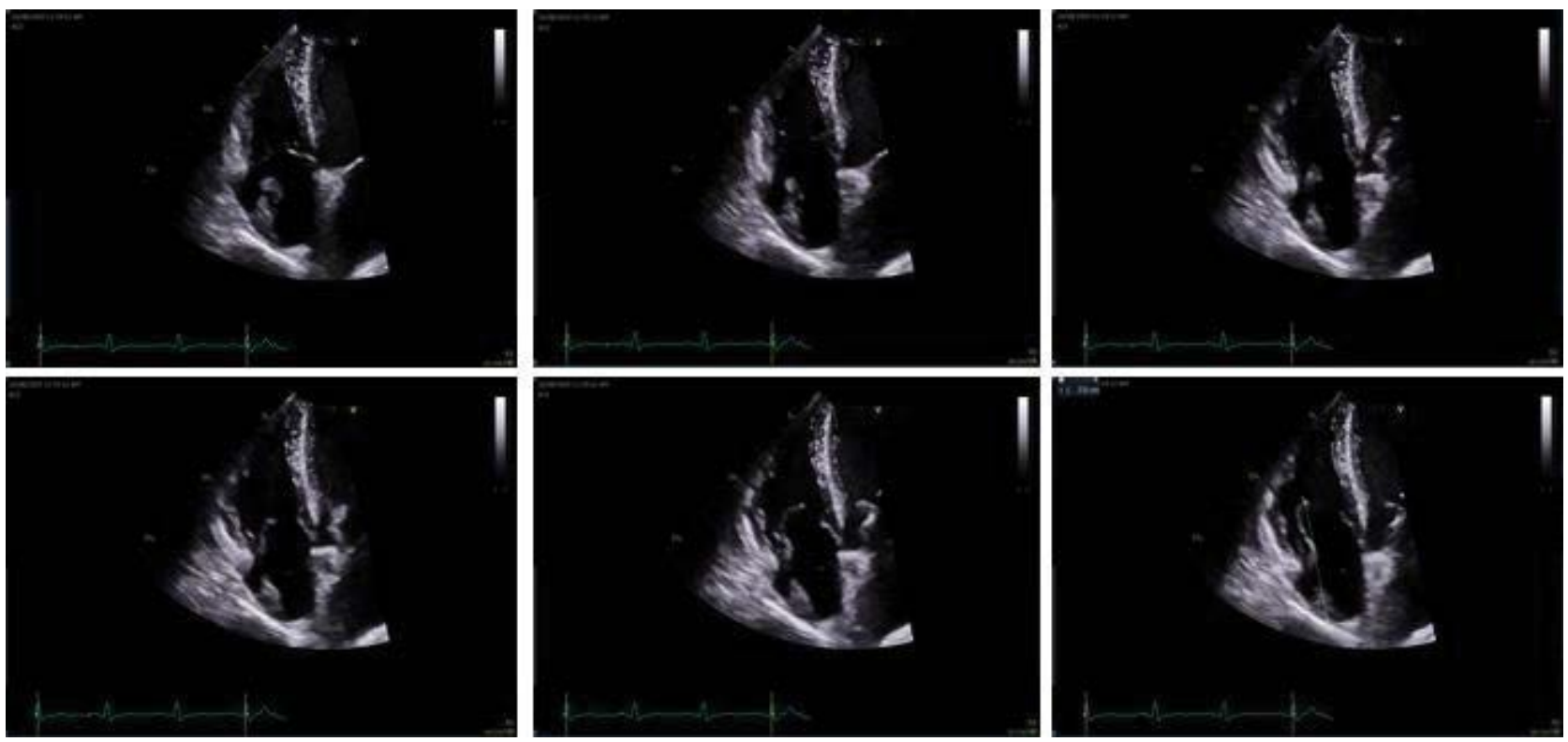

Figure 2: Transthoracic echocardiography demonstrating a large mass originating at the superior right atrial wall and inferior vena cava ostium that elongates into a highly mobile strand extending and prolapsing into the right ventricle in diastole.

mogenous mass measuring over $5.0 \mathrm{~cm}$ in length attached by a thick stalk to the superior right atrium and IVC junction. The mass prolapsed freely into the right ventricle in diastole (Figure 3). No other echocardiographic evidence of valvular heart disease was seen. Blood cultures drawn on admission remained negative and the patient remained a afebrile throughout the hospital course.

\section{Management (medical/interventions)}

Considering the echocardiographic findings, a heparin drip was initiated to prevent thromboembolic complications. Cardiothoracic surgery and cardiology were consulted. A multidisciplinary team decision to perform a percutaneous vacuum-assisted thrombectomy with the AngioVac device and venous chemo-port removal was made. An AngioVac cardiotomy resection of the right-sided intracardiac mass was performed utilizing venous-venous extracorporeal membrane oxygenation (ECMO) cardiopulmonary bypass under fluoroscopy and TEE guidance. The mobile portion of the mass was removed, accounting for approximately $80 \%$ of the lesion 

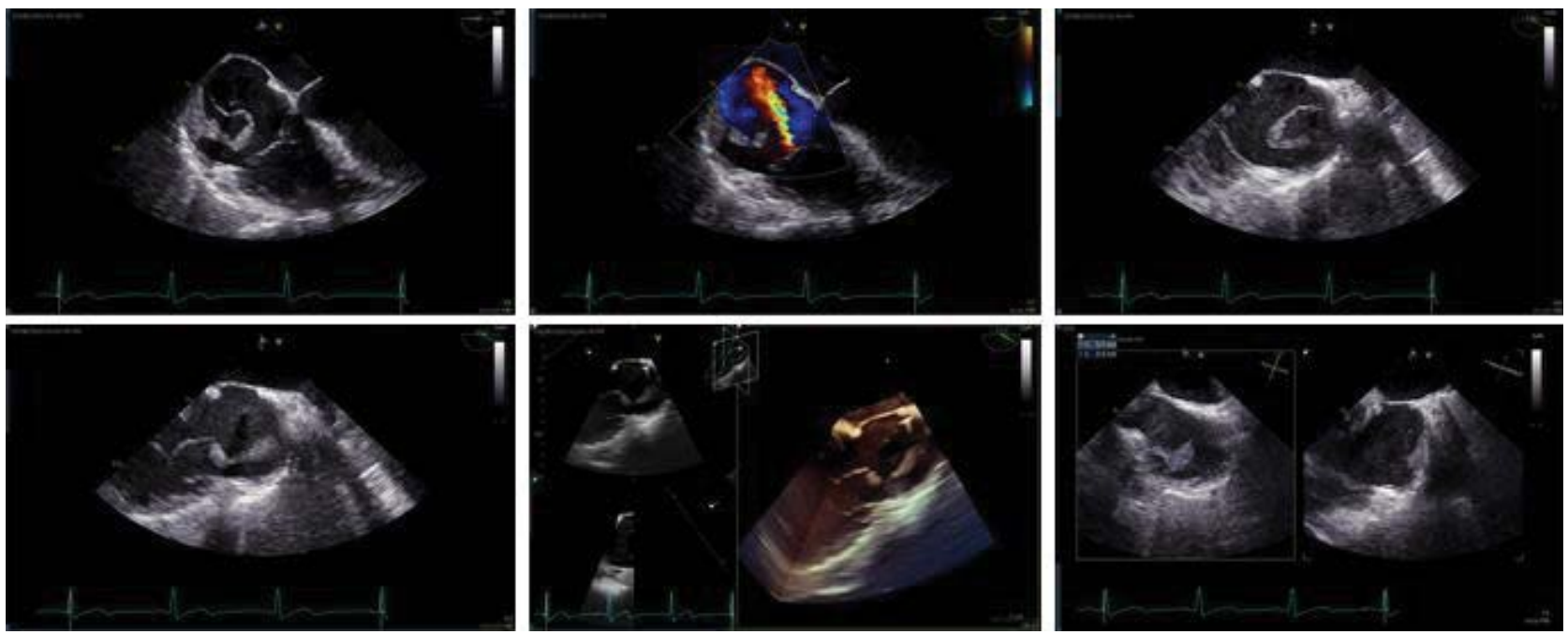

Figure 3: $2 \mathrm{D}$ and 3D transesophageal echocardiography demonstrating a large homogenous mass originating at the superior right atrial wall and inferior vena cava junction that extends and prolapses freely into the right ventricle during diastole.

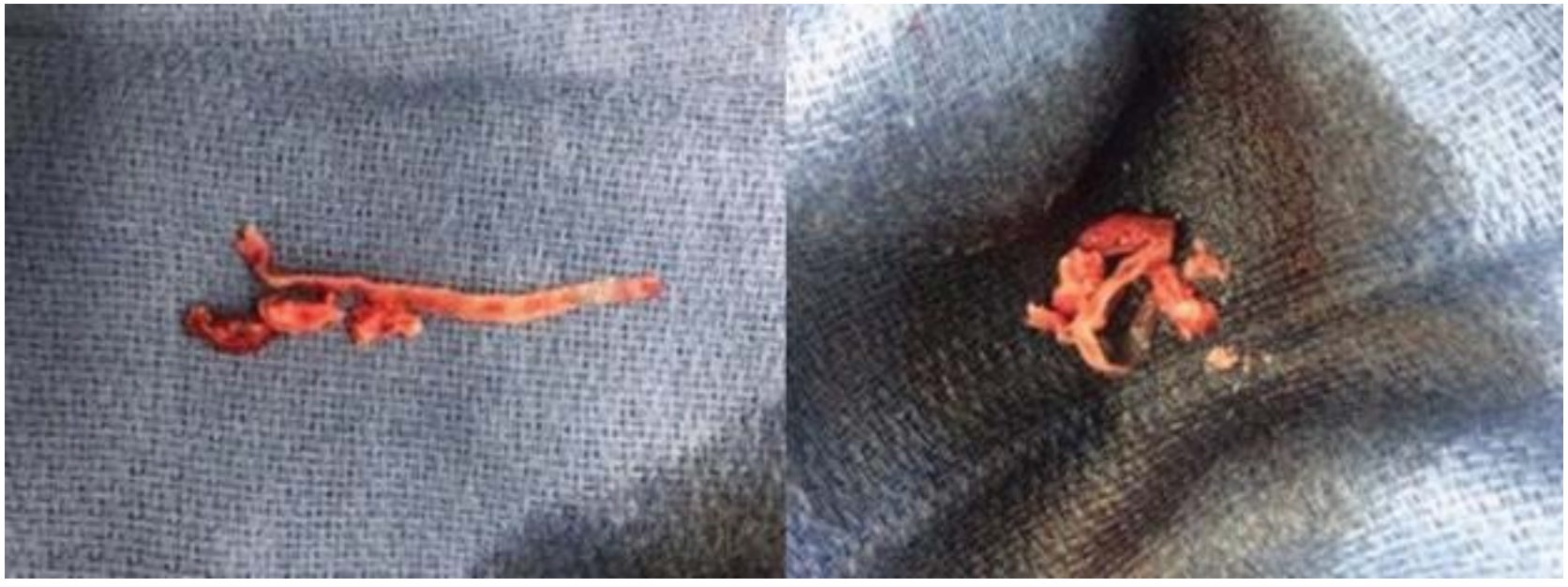

Figure 4: Gross imaging of the removed mobile portion of the intracardiac mass, which accounted for approximately $80 \%$ of the lesion.

(Figure 4). The sessile portion of the mass was unable to be extracted with the AngioVac system, as it brushed against the right atrial wall. Pathology results demonstrated decolorized thrombus with focal features of an early organization. There were no intra- or post-procedural complications and the patient was discharged on dabigatran for anticoagulation.

\section{Discussion}

Patients diagnosed with cancer often require placement of long-term access with central venous catheters for chemotherapy and administration of intravenous medications. Catheter- related thrombosis is a known complication of implantable venous access ports, with an incidence of $3.8 \%$. Regardless, primary thromboprophylaxis is currently not recommended in patients undergoing chemo-port placement [1]. Clinical presentation of catheter-related thrombosis varies greatly, ranging from asymptomatic to massive pulmonary embolism with associated hemodynamic instability [2]. While this patient presented with progressive dyspnea, imaging studies did not reveal any evidence of pulmonary embolism. Instead, a large, highly mobile, right atrial mass was observed by transthoracic echocardiogram. A transesophageal echocardiogram is often needed for further evaluation, and characterization of right atrial thrombi which is commonly associated with venous thromboembolic events [3].

Right heart thrombi are divided in two major subgroups by morphology, etiology, and clinical significance. Type A is often associated with a higher incidence of deep venous thrombosis, mostly originating from the peripheral veins and traveling into the right heart. On imaging studies, type $A$ thrombi are often described as a "worm-shape" appearance, and this category carries a high risk for the development of pulmonary embolism. Type B thrombi, develop within the right atrial cavity and are associated with atrial fibrillation and right-sided valvular disease, often resembling left ventricular thrombi in morphology, as they are often less mobile $[4,5]$. Therapeutic options often involve surgical embolectomy, sys- 
temic anticoagulation, and thrombolytic therapy. Recently, percutaneous vacuum-assisted thrombectomy utilizing the AngioVac aspiration system has been introduced as a feasible option for the removal of intravascular material (i.e. thrombus, masses, or vegetations) [6-8]. The AngioVac aspiration system is composed of a $22 \mathrm{~F}$ cannula with a suction capacity, extracorporeal circulatory system with an integrated filter, and a catheter to reinfuse the blood back to the patient. The system allows for the filtration of solid material within blood such as thrombus, foreign bodies, vegetation, and tumor [6]. A case series including 16 patients having undergone vacuum-assisted thrombectomy utilizing the Angiovac aspiration system demonstrated success in complete removal of the lesion. Indications for the procedure included right-sided intracardiac mass, catheter-associated thrombus, and caval thrombus. Most patients in the series (81.3\%), achieved complete removal of the lesion, while $12.5 \%$ achieved partial success and $6.3 \%$ were unsuccessful [6]. Similarly, our patient was found to have a catheter-associated thrombus within the superior right atrium and IVC junction which was treated utilizing the Angiovac aspiration system, with no intra-hospital complications.

\section{Follow-up}

Three weeks after hospital discharge, the patient was seen outpatient by cardiothoracic surgery. Patient denied any chest pain or shortness of breath and physical exam was unremarkable. Anticoagulation with dabigatran was continued to complete 6 months of therapy.

\section{Conclusion}

Right atrium thrombi are an uncommon complication of an implantable venous catheter. A transthoracic echocardiogram is a vital diagnostic tool in the evaluation of intracardiac lesions. However, a transesophageal echocardiogram is more reliable for the visualization and characterization of right-sided heart lesions. The AngioVac system has been proven to be an important and safe tool for the removal of intracardiac thrombus or mass. This case describes a patient presenting with shortness of breath attributed to a right atrial thrombus secondary to an implantable venous catheter. The patient was treated partially with percutaneous vacuum-assisted thrombectomy using the AngioVac system.

\section{Learning Objectives}

- Percutaneous Vacuum-assisted thrombectomy utilizing the AngioVac system is a safe and feasible option for removal of right-sided intracardiac lesions.
- Implanted venous catheters are a risk factor for the development of thromboembolic complications, including right atrial thrombus and non-bacterial thrombotic endocarditis.

- Clinical manifestation of thromboembolic complications' ranges from massive pulmonary embolism with hemodynamic instability to mild shortness of breath with no evidence of thromboembolic events.

\section{Conflicts of Interest}

The authors declared no potential conflicts of interest with respect to the research, authorship, and/or publication of this article.

\section{Funding}

The authors received no financial support for the research, authorship, and/or publication of this article.

Our institution does not require ethical approval for reporting individual cases or case series. Informed consent for patient information to be published in this article was not obtained because it was not required by our institution.

\section{References}

1. Decousus H, Bourmaud A, Fournel P, et al. (2018) Cancer-associated thrombosis in patients with implanted ports: A prospective multicenter French cohort study (ONCOCIP). Blood 132: 707716.

2. Dridi M, Mejri N, Labidi S, et al. (2016) Implantable port thrombosis in cancer patients: A monocentric experience. Cancer Biol Med 13: 384-388.

3. Ragland MM, Tak T (2006) The role of echocardiography in diagnosing space-occupying lesions of the heart. Clin Med Res 4: 22-32.

4. Kronik G (1989) The European Cooperative Study on the clinical significance of right heart thrombi. Eur Heart J 10: 1046-1059.

5. Sheikh O, Gulick D, Saenger E, et al. (2019) Management of right atrial thrombus. Where's the evidence. Curr Cardiol Rev 15.

6. Rajput FA, Du L, Woods M, et al. (2020) Percutaneous vacuum-assisted thrombectomy using angioVac aspiration system. Cardiovasc Revascularization Med 21: 489-493.

7. Smith SJ, Behrens G, Sewall LE, et al. (2014) Vacuum-assisted thrombectomy device (angiovac) in the management of symptomatic iliocaval thrombosis. J Vasc Interv Radiol 25: 425-430.

8. Shmueli $\mathrm{H}$, Thomas F, Flint N, et al. (2020) Right-sided infective endocarditis 2020: Challenges and updates in diagnosis and treatment. J Am Heart Assoc 9: e017293.

DOI: $10.36959 / 582 / 429$ 